\title{
THE AUTOMORPHISM GROUP OF A HOMOGENEOUS ALMOST COMPLEX MANIFOLD $\left(^{1}\right)$
}

\author{
BY \\ JOSEPH A. WOLF
}

1. Introduction. Let $M$ be a compact simply connected manifold of nonzero Euler characteristic that carries a homogeneous almost complex structure. We determine the largest connected group $A_{0}(M)$ of almost analytic automorphisms of $M$.

Our hypotheses represent $M$ as a coset space $G / K$ where $G$ is a maximal compact subgroup of the Lie group $A_{0}(M)$ and $K$ is a closed connected subgroup of maximal rank in $G$. In $\S 2$ we collect some information, decomposing $M=M_{1} \times \cdots \times M_{t}$ as a product of "irreducible" factors along the decomposition of $G$ as a product of simple groups; then every invariant almost complex structure or riemannian metric decomposes and every invariant riemannian metric is hermitian relative to any invariant almost complex structure; furthermore the decomposition is independent of $G$ in a certain sense. In $\$ 3$ we choose an invariant riemannian metric and determine the largest connected groups $\boldsymbol{H}_{0}\left(M_{i}\right)$ of almost hermitian isometries of the $M_{i}$. Then $A_{0}(M)$ is determined in $\S 4$. There it is shown that $A_{0}(M)=A_{0}\left(M_{1}\right)$ $\times \cdots \times A_{0}\left(M_{t}\right)$, that $A_{0}\left(M_{i}\right)=H_{0}\left(M_{i}\right)$ if the almost complex structure on $M_{i}$ is not integrable, and that $A_{0}\left(M_{i}\right)=H_{0}\left(M_{i}\right)^{C}$ if the almost complex structure on $M_{i}$ is induced by a complex structure. $\boldsymbol{A}_{0}(M)$ thus is a centerless semisimple Lie group whose simple normal analytic subgroups are just the $A_{0}\left(M_{i}\right)$.

2. Decomposition. Let $M$ be an effective coset space of a compact connected Lie group $G$ by a connected subgroup $K$ of maximal rank. In other words $M=G / K$ is compact, simply connected and of nonzero Euler characteristic; $G$ is a compact centerless semisimple Lie group, rank $K=\operatorname{rank} G$, and $K$ contains no simple factor of $G$. Then

$$
G=G_{1} \times \cdots \times G_{t}, \quad K=K_{1} \times \cdots \times K_{t} \quad \text { and } \quad M=M_{1} \times \cdots \times M_{t}
$$

where

$$
G_{i} \text { is simple, } \quad K_{i}=K \cap G_{i} \text { and } M_{i}=G_{i} / K_{i} .
$$

$G_{i}$ is a compact connected centerless simple Lie group, $K_{i}$ is a connected subgroup of maximal rank, and $M_{i}=G_{i} / K_{i}$ is a simply connected effective coset space of nonzero Euler characteristic. The decomposition of $M$ is unique up to order of the factors because it is determined by the decomposition of $G$.

Received by the editors November 5, 1966 and, in revised form, March, 1, 1969.

(1) Research partially supported by N.S.F. Grants GP-5798 and GP-8008. 
We call (2.1) the canonical decomposition of the coset space $M=G / K$. The factors $M_{i}=G_{i} / K_{i}$ are the irreducible factors of $M=G / K$. If there is just one irreducible factor, i.e. if $G$ is simple, then we say that $M=G / K$ is irreducible.

2.2. Proposition. Let $M$ be an effective coset space $G / K$ where $G$ is a compact connected Lie group and $K$ is a connected subgroup of maximal rank. Let $M=M_{1}$ $\times \cdots \times M_{t}$ be the canonical decomposition into irreducible factors $M_{i}=G_{i} / K_{i}$.

1. The G-invariant almost complex structures $J$ on $M$ are just the $J_{1} \times \cdots \times J_{t}$ where $J_{i}$ is a $G_{i}$-invariant almost complex structure on $M_{i}$.

2. The G-invariant riemannian metrics $d s^{2}$ on $M$ are just the $d s_{1}^{2} \times \cdots \times d s_{t}^{2}$ where $d s_{i}^{2}$ is a $G_{i}$-invariant riemannian metric on $M_{i}$; there each $\left(M_{i}, d s_{i}^{2}\right)$ is an irreducible riemannian manifold, so

$$
\left(M, d s^{2}\right)=\left(M_{1}, d s_{1}^{2}\right) \times \cdots \times\left(M_{t}, d s_{t}^{2}\right)
$$

is the de Rham decomposition.

3. Let $J$ be a $G$-invariant almost complex structure on $M$. If $d s^{2}$ is a G-invariant riemannian metric, then it is the real part of a G-invariant almost hermitian (for $J$ ) metric $h$ on $M$, and $h=h_{1} \times \cdots \times h_{t}$ where $h_{i}$ is a $G_{i}$-invariant almost hermitian ( for $J_{i}$ ) metric on $M_{i}$ and $d s_{i}^{2}$ is the real part of $h_{i}$.

Proof. The Lie algebras decompose uniquely as direct sums $\mathfrak{S}=\mathfrak{\Omega}+\mathfrak{M}$ and $\mathfrak{S}_{i}=\mathfrak{N}_{i}+\mathfrak{M}_{i}, \mathfrak{R}=\sum \mathfrak{R}_{i}$ and $\mathfrak{M}=\sum \mathfrak{M}_{i}$, with $[\mathfrak{R}, \mathfrak{M}] \subset \mathfrak{M}$ and $\left[\mathfrak{I}_{i}, \mathfrak{M}_{i}\right] \subset \mathfrak{M}_{i}$. Let $Z$ be the center of $K$, so $\Re$ is the centralizer of $Z$ in $\mathscr{S}$. Then $Z=Z_{1} \times \cdots \times Z_{t}$ where $Z_{i}$ is the center of $K_{i}$ and $\AA_{i}$ is the centralizer of $Z_{i}$ in $\mathscr{S}_{i}$.

$\pi$ denotes the representation of $K$ on $\mathfrak{M}$ and $\pi_{i}$ is the representation of $K_{i}$ on $\mathfrak{M}_{i}$. Then $\pi=\pi_{1} \oplus \cdots \oplus \pi_{t}$. Let $X=X_{1} \cup \cdots \cup X_{t}$ be the set of nontrivial characters on $Z$ such that

$$
\mathfrak{M}^{C}=\sum_{X} \mathfrak{M}_{x} \quad \text { and } \quad \mathfrak{M}_{i}^{C}=\sum_{X_{i}} \mathfrak{M}_{x}
$$

where $Z$ acts on $\mathfrak{M}_{\chi}$ by the character $\chi$. Each $\mathfrak{M}_{\chi}$ is ad $(K)$-stable, so $K$ acts on $\mathfrak{M}_{\chi}$ by a representation $\pi_{x}$, and

$$
\pi^{C}=\sum_{X} \pi_{x} \text { and } \pi_{i}^{C}=\sum_{X_{i}} \pi_{x}
$$

The point [7, Theorem 8.13.3] is that

$$
\text { the } \pi_{\chi} \text { are irreducible and mutually inequivalent. }
$$

We transform the complex decomposition (2.3) to a real decomposition. Let $X=S \cup T, S=S_{1} \cup \cdots \cup S_{t}$ and $T=T_{1} \cup \cdots \cup T_{t}$ where $S_{i}$ consists of the nonreal characters in $X_{i}$ and $T_{i}$ consists of the real ones. By real partition of $X$ we mean a disjoint $X=S^{\prime} \cup S^{\prime \prime} \cup T$ where $S^{\prime \prime}=\bar{S}^{\prime}$. If $\chi \in S_{i}$ then $\bar{\chi} \in S_{i}$; thus the real partition 
induces real partitions $X_{i}=S_{i}^{\prime} \cup S_{i}^{\prime \prime} \cup T_{i}$. If $|S|=2 n$ then $X$ has $2^{n}$ real partitions. Now choose a real partition $X=S^{\prime} \cup S^{\prime \prime} \cup T$ and define

$$
\begin{array}{ll}
\chi \in S^{\prime}: K \text { acts on } \mathfrak{M}_{\chi}^{R}=\mathfrak{M} \cap\left(\mathfrak{M}_{\chi}+\mathfrak{M}_{\bar{\chi}}\right) & \text { by } \pi_{\chi}^{R} \\
\chi \in T: K \text { acts on } \mathfrak{M}_{\chi}^{R}=\mathfrak{M} \cap \mathfrak{M}_{\chi} & \text { by } \pi_{\chi}^{R} .
\end{array}
$$

Then (2.3abc) becomes

$$
\begin{aligned}
\mathfrak{M} & =\sum_{S^{\prime}} \mathfrak{M}_{\chi}^{R}+\sum_{T} \mathfrak{M}_{\chi}^{R} \quad \text { and } \quad \mathfrak{M}_{i}=\sum_{S_{i}^{\prime}} \mathfrak{M}_{\chi}^{R}+\sum_{T_{i}} \mathfrak{M}_{\chi}^{R}, \\
\pi & =\sum_{S^{\prime}} \pi_{\chi}^{R}+\sum_{T} \pi_{\chi}^{R} \quad \text { and } \quad \pi_{i}=\sum_{S_{i}^{\prime}} \pi_{\chi}^{R}+\sum_{T_{i}} \pi_{\chi}^{R}
\end{aligned}
$$

the $\pi_{x}^{R}$ are real-irreducible and mutually inequivalent.

Let $\boldsymbol{A}$ be the commuting algebra of $\pi$ on $\mathfrak{M}$. By (2.4c), $\boldsymbol{A}=\sum_{\mathcal{S}^{\prime}} \boldsymbol{C}+\sum_{T} \boldsymbol{R}$, for $\pi_{\chi}^{R}$ has commuting algebra $\boldsymbol{C}$ if $\chi \in S^{\prime}, \boldsymbol{R}$ if $\chi \in T$. Invariant almost complex structures are in obvious correspondence with elements of square $-I$ of the commuting algebra, which now are seen to exist if and only if $T$ is empty, and (1) follows. Similarly, the decomposition of $d s^{2}$ in (2), and the existence and decomposition of $h$ in (3), are immediate.

It remains only to show the $\left(M_{i}, d s_{i}^{2}\right)$ irreducible as riemannian manifolds in (2). That fact is known $[3, \S 5.1]$, but in our present context we can give a short proof for the convenience of the reader. If $\left(M_{i}, d s_{i}^{2}\right)$ reduces, then it is a riemannian product $M^{\prime} \times M^{\prime \prime}$ because it is complete and simply connected, so we have an ad $\left(K_{i}\right)$-stable decomposition $\mathfrak{M}_{i}=\mathfrak{M}^{\prime} \oplus \mathfrak{M}^{\prime \prime}$ with the properties

$$
\left[\mathfrak{M}^{\prime}, \mathfrak{M}^{\prime \prime}\right] \subset \mathfrak{R}_{i}, \quad \mathfrak{M}^{\prime C}=\sum_{X^{\prime}} \mathfrak{M}_{x}, \quad \mathfrak{M}^{\prime \prime C}=\sum_{X^{\prime \prime}} \mathfrak{M}_{x}, \quad X_{i}=X^{\prime} \cup X^{\prime \prime} .
$$

Here $X^{\prime}$ and $X^{\prime \prime}$ are disjoint and self conjugate. If $\chi^{\prime} \in X^{\prime}$ and $\chi^{\prime \prime} \in X^{\prime \prime}$ with $\left[\mathfrak{M}_{\chi^{\prime}}, \mathfrak{M}_{\chi^{\prime \prime}}\right] \neq 0$, then $\chi^{\prime} \chi^{\prime \prime}=1$ so $\chi^{\prime}=\bar{\chi}^{\prime \prime} \in X^{\prime \prime}$ which is absurd. Thus $\left[\mathfrak{M}^{\prime}, \mathfrak{M}^{\prime \prime}\right]=0$, and it follows that the simple Lie algebra $\mathbb{B S}_{i}$ is direct sum of ideals

$$
\mathfrak{B S}^{\prime}=\left\{\mathfrak{\Re}_{i} \cap\left[\mathfrak{M}^{\prime}, \mathfrak{M}^{\prime}\right]\right\}+\mathfrak{M}^{\prime} \quad \text { and } \quad \mathfrak{B S}^{\prime \prime}=\left\{\mathfrak{\Re}_{i} \cap\left[\mathfrak{M}^{\prime \prime}, \mathfrak{M}^{\prime \prime}\right]\right\}+\mathfrak{M}^{\prime \prime} \text {. }
$$

That being absurd, irreducibility is proved. Q.E.D.

2.5. Remark. In the notation of the proof of Proposition 2.2, $M$ has a $G$ invariant almost complex structure if and only if $X=S$, and then those structures $J$ correspond to the real partitions $X=S^{\prime} \cup S^{\prime \prime}$ by: $\sum_{S^{\prime}} \mathfrak{M}_{x}$ and $\sum_{S^{\prime \prime}} \mathfrak{M}_{x}$ are the $\sqrt{ }-1$ and $-\sqrt{ }-1$ eigenspaces of $J$ on $\mathfrak{M}^{C}$.

3. Almost hermitian isometries. Let $M$ be a manifold with an almost hermitian metric $h$. Then $h=d s^{2}+(-1)^{1 / 2} \omega$ where the riemannian metric $d s^{2}$ is the real part of $h$ and $\omega(u, v)=d s^{2}(u, J v)$ is the imaginary part; that determines the almost complex structure $J$. By almost hermitian isometry of $(M, h)$ we mean a diffeomorphism that preserves $h$, i.e. that is a riemannian isometry of $\left(M, d s^{2}\right)$ which preserves $J$. 
Let $I(M)$ denote the (Lie) group of all isometries of $\left(M, d s^{2}\right), H(M)$ the closed subgroup consisting of those isometries that preserve $J$. Then $\boldsymbol{H}(M)$ is the (Lie) group of all almost hermitian isometries of $(M, h)$. In particular its identity component $\boldsymbol{H}_{0}(M)$ is an analytic subgroup of the identity component $\boldsymbol{I}_{0}(M)$ of $\boldsymbol{I}(M)$. If $(M, h)=\left(M_{1}, h_{1}\right) \times \cdots \times\left(M_{t}, h_{t}\right)$ hermitian product, then the de Rham decomposition says that $I_{0}(M)$ preserves each noneuclidean factor, so those factors are stable under $\boldsymbol{H}_{0}(M)$.

Let $M=G / K$ as in Proposition 2.2. Let $h$ be a $G$-invariant almost hermitian metric on $M$. The canonical decomposition induces $(M, h)=\left(M_{1}, h_{1}\right) \times \cdots \times\left(M_{t}, h_{t}\right)$ hermitian product where each $\left(M_{i}, d s_{i}^{2}\right), d s_{i}^{2}=\operatorname{Re} h_{i}$, is an irreducible noneuclidean riemannian manifold. Thus $\boldsymbol{H}_{0}(M)=\boldsymbol{H}_{0}\left(M_{1}\right) \times \cdots \times \boldsymbol{H}_{0}\left(M_{t}\right)$, and $\boldsymbol{H}(M)$ is generated by its subgroup $\boldsymbol{H}\left(M_{1}\right) \times \cdots \times \boldsymbol{H}\left(M_{t}\right)$ and permutations of mutually isometric $\left(M_{i}, h_{i}\right)$; so its determination is more or less reduced to the case where $M=G / K$ is irreducible. There the result is

3.1. Proposition. Let $M$ be an effective coset space $G / K$ where $G$ is a compact connected simple Lie group and $K$ is a connected subgroup of maximal rank. Let $h$ be a $G$-invariant almost hermitian metric on $M$, so $M=H_{0}(M) / B$ where $G \subset H_{0}(M)$ and $B \cap G=K$. If $G \neq H_{0}(M)$, then $(M, h)$ is an irreducible hermitian symmetric space of compact type listed below.

\begin{tabular}{|c|c|c|c|c|c|}
\hline Case & $G$ & $K$ & $H_{0}(M)$ & $B$ & $(M, h)$ \\
\hline 1 & $G_{2}$ & $U(2)$ & $S O(7)$ & $S O(5) \times S O(2)$ & $\begin{array}{l}\text { 5-dimensional } \\
\text { complex quadric }\end{array}$ \\
\hline 2 & $S p(r) / Z_{2}$ & $S_{p(r-1) \cdot U(1)}$ & $S U(2 r) / Z_{2 r}$ & $U(2 r-1)$ & $\begin{array}{l}\text { complex projective } \\
(2 r-1) \text {-space }\end{array}$ \\
\hline 3 & $S O(2 r+1)$ & $U(r)$ & $S O(2 r+2) / Z_{2}$ & $U(r+1) / Z_{2}$ & $\begin{array}{l}\text { unitary structures } \\
\text { on } R^{2 r+2}\end{array}$ \\
\hline $3^{\prime}$ & $S p i n(7) / Z_{2}$ & $U(3)$ & $S O(8) / Z_{2}$ & $S O(6) \cdot S O(2)$ & $\begin{array}{l}\text { 6-dimensional } \\
\text { complex quadric }\end{array}$ \\
\hline
\end{tabular}

REMARK 1. In the exceptional cases above, $K$ is not $\boldsymbol{R}$-irreducible on the tangent space, so $M$ has another $G$-invariant almost hermitian metric for which $G=H_{0}(M)$.

REMARK 2. The proof is easily reduced to the case where $B$ is the centralizer of a toral subgroup of $\boldsymbol{H}_{0}(M)$, and then the result can be extracted from [2, Table 5] and the Bott-Borel-Weil Theorem. But here it is convenient to reduce the proof to some classifications of Oniščik [4].

Proof. As $M$ has nonzero Euler characteristic, $B$ has maximal rank in $H_{0}(M)$, so $H_{0}(M) / B=G / K$ is one of the following entries in Oniščik's list [4, Table 7].

(i) $A_{2 n-1} / A_{2 n-2} \cdot T=C_{n} / C_{n-1} \cdot T$ (our Case 2),

(ii) $B_{3} / B_{2} \cdot T=G_{2} / A_{1} \cdot T$ (our Case 1 ),

(iii) $B_{3} / D_{3}=G_{2} / A_{2}\left(B_{3}\right.$ does not preserve $J$ here), 
(iv) $D_{n+1} / A_{n} \cdot T=B_{n} / A_{n-1} \cdot T$ (our Case 3 ),

(v) $D_{4} / D_{3} \cdot T=B_{3} / A_{2} \cdot T$ (our Case $3^{\prime}$ ).

The assertions follow with the observation that $\boldsymbol{H}_{0}(M) / B$ is an irreducible hermitian symmetric coset space of compact type in each of the admissible cases. Q.E.D.

4. Almost analytic automorphisms. Let $M$ be a manifold with almost complex structure $J$. By almost analytic automorphism of $M$, we mean a diffeomorphism of $M$ which preserves $J$. The set of all such diffeomorphisms forms a group $A(M)$. If $M$ is compact, then [1] in the compact-open topology, $A(M)$ is a Lie transformation group of $M$. We denote its identity component by $A_{0}(M)$. If, further, we have an almost hermitian metric on $M$, then $H(M)$ is a compact subgroup of $A(M)$. That will be our main tool in studying $A(M)$.

4.1. THEOREM. Let $M=G / K$ be a simply connected effective coset space of nonzero Euler characteristic where $G$ is a compact connected Lie group. Let $J$ be a $G$-invariant almost complex structure on $M$. Let $M=M_{1} \times \cdots \times M_{t}$ be the canonical decomposition into irreducible coset spaces, and decompose $J=J_{1} \times \cdots \times J_{t}$ where $J_{i}$ is a $G_{i}$-invariant almost complex structure on $M_{i}$. Then

1. $A_{0}(M)=A_{0}\left(M_{1}\right) \times \cdots \times A_{0}\left(M_{t}\right)$.

2. $M$ has a G-invariant riemannian metric $d s^{2}=d s_{1}^{2} \times \cdots \times d s_{t}^{2}$ for which $\boldsymbol{H}_{0}(M)$ is a maximal compact subgroup of $A_{0}(M)$.

3. If $J_{i}$ is integrable then $A_{0}\left(M_{i}\right)=H_{0}\left(M_{i}\right)^{C}$. If $J_{i}$ is not integrable then $A_{0}(M)=$ $H_{0}(M)$.

Proof. For the second statement, enlarge $G$ to a maximal compact subgroup $H$ of $A_{0}(M)$ and choose an $H$-invariant riemannian metric $d s^{2}$ on $M$. Then $d s^{2}$ $=d s_{1}^{2} \times \cdots \times d s_{t}^{2}$ as required, by Proposition 2.2 , and $H=H_{0}(M)$ by construction.

We simplify notation for the proofs of the first and third statement by enlarging $G$ to $H_{0}(M)$ and writing $A$ for $A_{0}(M)$. That does not change the canonical decomposition of $M$, for the latter is the de Rham decomposition for $d s^{2}$ according to Proposition 2.2. Now $G / K=M=A / B$ where $G \subset A$ is a maximal compact subgroup and $K=G \cap B$.

We check that $A$ is a centerless semisimple Lie group. If $L$ is a closed normal analytic subgroup of $A$ with $G \cap L$ discrete, then $G \cdot L \subset A$ is effective on

$$
(G \cdot L) /(K \cdot L)=M, \text { so } L=\{1\} .
$$

Let $L$ be the radical of $A$ : now $A$ is semisimple. Let $\mathfrak{L}$ be the orthocomplement of $\mathfrak{B S}$ in a maximal compactly embedded subalgebra of $\mathfrak{A}$ : now $A$ has finite center, so the centerless group $G$ contains the center of $A$, so $A$ is centerless.

Let $A^{\alpha}, 1 \leqq \alpha \leqq r$, be the simple normal analytic subgroups of $A$. So $A=A^{1} \times \cdots$ $\times A^{r}$ with $A^{\alpha}$ centerless simple. Now $G=G^{1} \times \cdots \times G^{r}, K=K^{1} \times \cdots \times K^{r}$ and $M=M^{1} \times \cdots \times M^{r}$ where

$$
G^{\alpha}=G \cap A^{\alpha}, \quad K^{\alpha}=K \cap G^{\alpha}, \quad M^{\alpha}=G^{\alpha} / K^{\alpha} .
$$


If $\alpha \neq \beta$ then $A^{\alpha}$ acts trivially on $M^{\beta}$. For every $a \in A^{\alpha}$ centralizes the transitive transformation group $G^{\beta}$ of $M^{\beta}$, hence induces some transformation $\bar{a}$ of $M^{\beta}$ that is trivial or fixed point free. As $A^{\alpha}$ is connected, $\bar{a}$ is homotopic to 1 so its Lefschetz number is the (nonzero) Euler characteristic of $M^{\beta}$; that shows $\bar{a}=1$. Now $M^{\alpha}=A^{\alpha} / B^{\alpha}, B^{\alpha}=B \cap A^{\alpha}$, with $B=B^{1} \times \cdots \times B^{r}$.

According to Oniščik [5, Table 1] the only possibilities for $G^{\alpha} / K^{\alpha}=M^{\alpha}=A^{\alpha} / B^{\alpha}$, $A^{\alpha}$ noncompact, are given in the following table.

\begin{tabular}{|c|c|c|}
\hline$A^{\alpha}$ & $M^{\alpha}=G^{\alpha} / K^{\alpha}$ & Conditions \\
\hline$S L(2 n, R) / Z_{2}$ & $\operatorname{SO}(2 n) / \operatorname{SO}\left(2 n_{1}\right) \times \cdots \times \operatorname{SO}\left(2 n_{p}\right)$ & $n=\sum n_{i}>1$ \\
\hline$S L(2 n+1, R)$ & $\operatorname{SO}(2 n+1) / S O\left(2 n_{1}\right) \times \cdots \times \operatorname{SO}\left(2 n_{p-1}\right) \times \operatorname{SO}\left(2 n_{p}+1\right)$ & $n=\sum n_{i}$ \\
\hline$G L(n, Q) / Z_{2}$ & $S p(n) / S p\left(n_{1}\right) \times \cdots \times S p\left(n_{p}\right) \times U(1)^{q}$ & $n=q+\sum n_{i}$ \\
\hline$S O(1,2 n-1) / Z_{2}$ & $\operatorname{SO}(2 n-1) / \operatorname{SO}\left(2 n_{1}\right) \times \cdots \times \operatorname{SO}\left(2 n_{p}\right) \times U\left(m_{1}\right) \times \cdots \times U\left(m_{q}\right)$ & $\begin{array}{l}n-1 \\
=\sum n_{i}+\sum m_{j}\end{array}$ \\
\hline$E_{6, c_{4}} / Z_{2}$ & $S p(4) / S p(2) \times S p(2)$ and $S p(4) /[S p(1)]^{4}$ & none \\
\hline$E_{6, F_{4}}$ & $F_{4} / \operatorname{Spin}(9), F_{4} / \operatorname{Spin}(8), F_{4} / U(4)$ and $F_{4} /[S U(2)]^{4}$ & none \\
\hline$\left(G^{\alpha}\right)^{C}$ & $\begin{array}{l}G^{\alpha} / K^{\alpha} \text { where } K^{\alpha} \text { is the centralizer of a nontrivial toral } \\
\text { subgroup of } G^{\alpha}\end{array}$ & $\begin{array}{l}G^{\alpha} \text { compact } \\
\text { centerless } \\
\text { simple }\end{array}$ \\
\hline
\end{tabular}

Note that $G^{\alpha}$ is simple except in Case 1 with $n=2$. There $M^{\alpha}$ is the product of two Riemann spheres, so $A^{\alpha}$ is the product of two copies of $S L(2, C) / Z_{2}$, contradicting the table entry for $A^{\alpha}$. Thus we always have $G^{\alpha}$ simple, so each $M^{\alpha}$ is an $M_{i}$, and the first statement of our theorem is proved with $A^{\alpha}=A_{0}\left(M^{\alpha}\right)$.

Now we may, and do, assume $M$ irreducible. Thus $A$ and $G$ are simple.

4.2. Lemma. The invariant almost complex structure $J$ is integrable if and only if $A=G^{C}$. In that case $B$ is a complex parabolic subgroup of $A$ and $J$ is induced either from the natural complex structure on $A / B$ or from the conjugate structure.

Proof of lemma. Let $J$ be integrable; we check $\left(^{C} \subset \subset \mathfrak{A}\right.$. For if $\xi \in \mathbb{B}$ and $\xi^{*}$ denotes the holomorphic vector field induced on " $M$, then $J\left(\xi^{*}\right)$ is holomorphic. Thus $\mathbb{S S}^{C}$ acts on $M$ by $\xi+i \eta \rightarrow \xi^{*}+J\left(\eta^{*}\right)$, and this action integrates to $G^{C}$ because $M$ is compact; that shows $G^{C} \subset A$ so $\mathbb{S}^{C} \subset \mathfrak{A}$.

Let $\mathfrak{A}=\mathbb{S S}^{C}$. As $\mathfrak{A}$ is its own normalizer in $\mathscr{B S}$ because it has maximal rank, $\mathfrak{B}$ is its own normalizer in $\mathfrak{A}$, so $B$ is an $\boldsymbol{R}$-algebraic subgroup of $A$. Thus $A$ has an Iwasawa decomposition $G S N$ with $B=K S N$. As $\mathfrak{A}=\mathscr{B S}^{C}$, the group $S^{C}$ is a complex Cartan subgroup of $A$, so $N$ is a complex unipotent subgroup. Now $K^{C} S^{C} N$ is the complex group generated by $B$ and it has intersection $K$ with $G$; thus $M=$ $A / B \rightarrow A / K^{C} S^{C} N=G / K$ is trivial so $B$ is a complex subgroup of $A$. As $A / B$ is compact now $B$ is a complex parabolic subgroup. 
Decompose $B=B^{r} \cdot B^{u}$ into reductive and unipotent parts. Let $Z$ be the identity component of the center of $B^{r}$, complex subtorus of $S^{C}$. Let $D$ be the set of characters $\chi \neq 1$ on $Z$ that are restrictions of positive roots, sQ $\mathfrak{B}^{u}=\sum_{D} \mathfrak{A}_{\chi}$. Define $\mathfrak{B}^{-u}=\sum_{D} \mathfrak{A}_{-x}$ so that $\mathfrak{A}$ is the direct sum of its subspaces $\mathfrak{B}^{r}, \mathfrak{B}^{u}$ and $\mathfrak{B}^{-u}$. (S) $\cap\left(\mathfrak{B}^{u}+\mathfrak{B}^{-u}\right)$ represents the real tangent space of $M$, and $\mathfrak{B}^{u}+\mathfrak{B}^{-u}$ represents the complexified tangent space. If $\pm \chi \in D$, then $\mathfrak{A}_{x}$ is an irreducible representation space of $B^{r}$, so $J$ acts on $\mathfrak{A}_{x}$ either as $\sqrt{ }-1$ or as $-\sqrt{ }-1$. Let $\mathfrak{L}^{+}$(resp. $\mathfrak{Q}^{-}$) denote the image in $\mathfrak{A} / \mathfrak{B}$ of the $\mathfrak{A}_{\chi},-\chi \in D$, on which $J$ acts as $\sqrt{ }-1$ (resp. $\left.-\sqrt{ }-1\right)$. Then ad (B) $\cdot \mathfrak{Q}^{ \pm} \subset \mathfrak{Q}^{ \pm}$by invariance of $J$ under $B$. If $\nu$ is the restriction to $Z$ of the highest root, then $\mathfrak{A} / \mathfrak{B}=\sum_{n \geqq 0}$ ad $(\mathfrak{B})^{n} \cdot\left(\mathfrak{A}_{-\nu} \bmod \mathfrak{B}\right)$, because $\mathfrak{A}$ is simple, so $\mathfrak{A} / \mathfrak{B}$ is the one of $\mathfrak{L}^{+}$or $\mathfrak{L}^{-}$into which $\mathfrak{A}_{-v}$ maps. Thus either $J$ acts on $\mathfrak{B}^{-u}$ as $\sqrt{ }-1$ and the natural complex structure of $A / B$ induces $J$, or $J$ acts on $\mathfrak{B}^{-u}$ as $-\sqrt{ }-1$ and the natural structure induces $-J$. In either case $J$ is integrable.

In general suppose $\mathbb{B S}^{C} \subset \mathfrak{A}$. Then $M=G^{C} / B \cap G^{C}$ is a complex flag manifold on which $A$ is the largest connected group of analytic automorphisms. Thus $A$ is a centerless complex semisimple group, hence the complexification of its maximal compact subgroup $G$.

Lemma 4.2 is proved.

4.3. Lemma. If $B^{C}$ is parabolic in $A^{C}$, then $J$ is integrable so $A=G^{C}$.

Proof of lemma. $J$ is an element of square $-I$ in the commuting algebra of ad $(\mathfrak{B})$ on $\mathfrak{A} / \mathfrak{B}$. Thus it induces an element $J^{C}$ of square $-I$ in the commuting algebra of ad $\left(\mathfrak{B}^{C}\right)$ on $\mathfrak{A}^{C} / \mathfrak{B}^{C}$. Now suppose $B^{C}$ parabolic in $A^{C}$, so $M^{C}=A^{C} / B^{C}$ is compact and of positive Euler characteristic with invariant almost complex structure $J^{C}$.

If $A$ is complex then $A=G^{C}$ and Lemma 4.2 says that $J$ is integrable. Thus we may assume $A$ not complex so that $A^{C}$ is simple. Then Lemma 4.2 says that $J^{C}$ is integrable, and in fact that either $J^{C}$ or $-J^{C}$ is induced by the natural complex structure on $A^{C} / B^{C}$. Replace $J$ by $-J$ if necessary; that does not alter integrability of $J$, but it replaces $J^{C}$ by $-J^{C}$, allowing us to assume $J^{C}$ induced by the natural complex structure of $A^{C} / B^{C}$.

Decompose $B=B^{r} \cdot B^{u}$ into reductive and unipotent parts, so $\mathfrak{B}=\mathfrak{B}^{r}+\mathfrak{B}^{u}$ and $\mathfrak{U}=\mathfrak{B}+\mathfrak{B}^{-u}$ where $\mathfrak{B}^{ \pm u}$ are subalgebras normalized by $\mathfrak{B}^{r}$. Let $\mathfrak{B}^{-u}$ represent the real tangent space to $M$. Note that $J^{C}$ acts on $\left(B^{-u}\right)^{C}$ as $\sqrt{ }-1$. That contradicts our arrangement that the action of $J^{C}$ on $\left(\mathfrak{B}^{-u}\right)^{C}$ is induced by the action of $J$ on $\mathfrak{B}^{-u}$. Thus $A$ cannot be noncomplex. Lemma 4.3 is proved.

We complete the proof of Theorem 4.1. As in the second paragraph of the proof of Lemma $4.2, B$ is a real algebraic subgroup of $A$, so there is a semidirect product decomposition $B=B^{r} \cdot B^{u}$ into reductive and unipotent parts. If rank $B^{r}<\operatorname{rank} A$, then any Cartan subalgebra of $\mathfrak{A}$ has an element $\xi$ not contained in any isotropy subalgebra of $\mathfrak{A}$ on $M$ so it induces a nonvanishing vector field $\xi^{*}$ on $M$. The 
existence of a nonvanishing vector field $\xi^{*}$ says that $M$ has Euler characteristic zero. That contradiction proves rank $B^{r}=\operatorname{rank} A$.

Let $\sigma$ be the Cartan involution of $\mathfrak{A}$ with fixed point set $\mathfrak{B S}$ and let $\mathfrak{A}=\mathbb{S}+\mathfrak{B}$ be the Cartan decomposition. We may assume $\sigma\left(\mathfrak{B}^{r}\right)=\mathfrak{B}^{r}$, so $\mathfrak{B}^{r}=\mathfrak{R}+\left(\mathfrak{B} \cap \mathfrak{B}^{r}\right)$. That gives compact real forms

$$
\mathfrak{A}_{c}=\mathfrak{S}+\sqrt{ }-1 \mathfrak{B} \quad \text { and } \quad \mathfrak{B}_{c}^{r}=\mathfrak{R}+\sqrt{ }-1\left(\mathfrak{P} \cap \mathfrak{B}^{r}\right) .
$$

Let $A_{c}$ denote the centerless group with Lie algebra $\mathfrak{A}_{c}$ and let $B_{c}^{r}$ be the analytic subgroup for $\mathfrak{B}_{c}^{r}$. Then rank $B_{c}^{r}=\operatorname{rank} B^{r}=\operatorname{rank} A=\operatorname{rank} A_{c}$ tells us that $X=A_{c} / B_{c}^{r}$ is a compact simply connected manifold of positive Euler characteristic. If $A=G$ then $B=B^{r}=K$, so $A_{c}=G$ and $B_{c}^{r}=K$, whence $X=M$.

As in the second paragraph of the proof of Lemma 4.2 we have Iwasawa decompositions $A=G S N$ and $B=K S N$. Choose a torus subgroup $T \subset K$ such that $H=T \times S \subset B^{r}$ is a Cartan subgroup of $A$. Let $\Delta$ be the root system. Now $\Delta=D \cup E$ $\cup-E$ disjoint, and $\mathfrak{A}=\mathfrak{B}^{r}+\mathfrak{B}^{u}+\mathfrak{B}^{-u}$ direct, where

$$
\mathfrak{B}^{r}=\mathfrak{S}+\mathfrak{A} \cap\left\{\sum_{D} \mathfrak{A}_{\phi}\right\}, \quad \mathfrak{B}^{u}=\mathfrak{A} \cap\left\{\sum_{E} \mathfrak{A}_{\phi}\right\}, \quad B^{-u}=\mathfrak{A} \cap\left\{\sum_{\boldsymbol{E}} \mathfrak{A}_{-\phi}\right\}
$$

Observe that $\sigma$ interchanges $\mathfrak{B}^{u}$ and $\mathfrak{B}^{-u}$. For $\mathfrak{B}^{u} \subset N$ because $N=N^{\prime} \cdot B^{u}$ where $B^{r}=K S N^{\prime}$, and the dual space of $\subseteq$ has an ordering such that

$$
\mathfrak{N}^{C}=\sum_{\phi \mid \varsigma>0} \mathfrak{A}_{\phi}, \text { and }\left.\phi\right|_{\odot}>0 \text { iff }\left.\sigma \phi\right|_{\odot}<0 .
$$

View the invariant almost complex structure $J$ of $M$ as an element of square $-I$ in the commuting algebra of ad $(\mathfrak{B})$ on $\mathfrak{A} / \mathfrak{B}$, hence in the commuting algebra of ad $\left(\mathfrak{B}^{r}\right)$ on $\mathfrak{B}^{-u} \cong \mathfrak{A} / \mathfrak{B}$; then extend $J$ to an element $J^{\prime}$ of square $-I$ in the commuting algebra of ad $\left(\mathfrak{B}^{r}\right)$ on $\mathfrak{B}^{u}+\mathfrak{B}^{-u}$ by the formula

$$
J^{\prime}(\xi+\eta)=\sigma J(\sigma \xi)+J(\eta) \quad \text { where } \xi \in \mathfrak{B}^{u}, \eta \in \mathfrak{B}^{-u} .
$$

Now $J^{\prime}$ is an $A$-invariant $\sigma$-invariant almost complex structure on $A / B^{r}$, so [6, Proposition 7.7] it defines an $A_{c}$-invariant $\sigma$-invariant almost complex structure on $A_{c} / B_{c}^{r}$. We have proved that $X=A_{c} / B_{c}^{r}$ has an invariant almost complex structure.

Suppose $A \neq G$. Note that [6, Theorem 4.10] eliminates lines 5 and 6 of the Oniščik table above, so either $A=G^{C}$ or $A$ is absolutely simple and of classical type. Suppose $A \neq G^{C}$ so $A_{c}$ is simple and of classical type. Then [6, Theorem 4.10] shows that $B_{c}^{r}$ is the centralizer of a torus in $A_{c}$. Let $\beta_{c}$ denote the center of $\mathfrak{B}_{c}^{r}$. Then $\sigma\left(\mathfrak{B}_{c}^{r}\right)=\mathfrak{B}_{c}^{r}$ implies $\sigma\left(\mathbb{B}_{c}\right)=\mathbb{Z}_{c}$, so $\mathbb{B}_{c}=\mathfrak{U}+(-1)^{1 / 2} \mathfrak{B}$ with $\mathfrak{U} \subset \mathfrak{A}$ and $\mathfrak{B} \subset \mathfrak{B} \cap \mathfrak{B}^{r}$. Now $\mathfrak{B}^{r}$ has center $\mathbb{B}=\mathfrak{U}+\mathfrak{B} \subset \mathfrak{I}+\mathfrak{S}=\mathfrak{S}$, and $\mathfrak{B}^{r}$ is the centralizer of 8 in $\mathfrak{A}$. We order the root system $\Delta$ so that a root $\phi>0$ whenever $\left.\phi\right|_{3} \neq 0$ and $\left.\phi\right|_{\odot}>0$. Then $\mathfrak{B}^{C}$ contains the Borel subalgebra $\mathfrak{S}^{C}+\sum_{\phi>0} \mathfrak{A}_{\phi}$ of $\mathfrak{A}^{C}$ for that ordering, so $\mathfrak{B}^{C}$ is a parabolic subalgebra of $\mathfrak{A}^{C}$. Then Lemma 4.3 says $A=G^{C}$. We have proved that $A \neq G$ implies $A=G^{C}$. 
If $J$ is integrable then Lemma 4.2 says $A=G^{C}$. If $J$ is not integrable then Lemma 4.2 says $A \neq G^{C}$, so we cannot have $A \neq G$, and that forces $A=G$. Theorem 4.1 is proved. Q.E.D.

4.3. Remark. Theorem 4.1 extends the scope of [8, Theorem 17.4(3)], but that result remains incomplete because, as remarked at the end of $[8, \S 17]$, it is not known whether

$$
A_{0}\left(\boldsymbol{E}_{6} / \operatorname{ad} \boldsymbol{S U}(3)\right) \text { is } \boldsymbol{E}_{6} \text { rather than } \boldsymbol{E}_{6}^{C}
$$

or whether

$$
\begin{aligned}
& A_{0}\left(S O\left(n^{2}-1\right) / \operatorname{ad} S U(n)\right) \text { is } S O\left(n^{2}-1\right) \text { rather than } \\
& S O\left(n^{2}-1, C\right), \quad S L\left(n^{2}-1, R\right), \text { or } S O\left(1, n^{2}-1\right) .
\end{aligned}
$$

\section{REFERENCES}

1. W. M. Boothby, S. Kobayashi and H.-C. Wang, A note on mappings and automorphisms of almost complex manifolds, Ann. of Math. 77 (1963), 329-334.

2. E. B. Dynkin, The maximal subgroups of the classical groups, Trudy Moskov. Mat. Obšč. 1 (1952), 39-166; Amer. Math. Soc. Transl. (2) 6 (1957), 245-378.

3. B. Kostant, On holonomy and homogeneous spaces, Nagoya Math. J. 12 (1957), 31-54.

4. A. L. Oniščik, Inclusion relations among transitive compact transformation groups, Trudy Moskov. Mat. Obšč. 11 (1962), 199-242; Amer. Math. Soc. Transl. (2) 50 (1966), 5-58.

5. —_, Lie groups transitive on compact manifolds. III, Mat. Sb. 75 (117) (1968), 255-263= Math. USSR Sbornik 4 (1968).

6. J. A. Wolf and A. Gray, Homogeneous spaces defined by Lie group automorphisms. I, J. Differential Geometry 2 (1968), 77-114; II, ibid. 115-159.

7. J. A. Wolf, Spaces of constant curvature, McGraw-Hill, New York, 1967.

8. - The geometry and structure of isotropy irreducible homogeneous spaces, Acta Math. 120 (1968), 59-148.

UNIVERSITY OF CALIFORNIA, Berkeley, CAlifornia 